\title{
Diagnosis of Regional Monthly Anomalies Using the Adjoint Method. Part II: Potential Vorticity
}

\author{
ANDREW W. ROBERTSON \\ Meteorologisches Institut der Universität München, Munich, Germany
}

(Manuscript received 23 January 1991, in final form 5 December 1991)

\begin{abstract}
An equivalent barotropic tracer model and its adjoint are used to examine observed central European anomalies of potential vorticity (PV) in terms of advection and diabatic effects. The PV viewpoint yields a similar picture of six January anomalies to that found using a one-layer tropospheric temperature model, with the main component of the largest anomalies resulting from advection of PV present at the beginning of the month. Remote PV plays a larger role due to more rapid advection at upper levels. Anomalous diabatic effects are estimated as a residual from the tracer model itself. They are found to be of similar importance for the PV anomalies as are the (residual) source terms in the one-layer thermodynamic equation in the tropospheric temperature case. However, the spatial fields of the residual monthly averaged diabatic effects are noisier and more difficult to interpret than in the temperature case. An interpretation in terms of an estimate of the cross-isentropic mass flux at the base of the model is attempted.
\end{abstract}

\section{Introduction}

In Part I of this paper (Robertson 1992) regional tropospheric temperature anomalies were investigated using the adjoint method of Marchuk (1974) applied to a simple one-layer temperature tracer model. The results demonstrate the ability of the adjoint of a onelayer model to provide a novel diagnostic picture of European temperature anomalies.

The aim of Part II is to carry out the same treatment for potential vorticity (PV) anomalies. In contrast to temperature, which is a passive tracer (making it difficult to link anomalous advection with the mechanisms maintaining the anomaly), the PV constitutes a dynamic tracer so that its advection has implications for the evolution of the flow and the dynamics maintaining a regional anomaly. For example, the closed PV contours associated with a blocking anomaly suggest a local dynamical maintenance (McWilliams 1980), whereas the opposite must necessarily be the case in a rapidly $\mathrm{PV}$-advecting situation. The complete description of the flow provided by the PV, whereby both flow and temperature fields can be derived from the PV field, provided boundary conditions, a reference state, and a balance condition are specified (the "invertibility principle," Hoskins et al. 1985) lends this variable added interest.

Observational evidence (Blackmon et al. 1979) indicates that monthly regional anomalies $(\sim 1000 \mathrm{~km})$ are characterized by equivalent barotropic vertical structures, so that a one-layer PV model provides a

Corresponding author address: Dr. A. W. Robertson, Dept. of Atmospheric Sciences, University of California, 405 Hilgard Avenue, Los Angeles, CA 90024-1565. natural first choice for an adjoint tracer study. An equivalent barotropic model of the potential vorticity has recently been discussed by Salby (1989). It describes column-averaged behavior, reflecting motions within about a scale height of some lower boundary, that is, "deep circulations where the motion field is vertically aligned over one or more scale heights" (Salby 1989). The vertical stratification is assumed to be "equivalent barotropic," so that two families of thermodynamic surfaces (e.g., $T$ and $p$ ) are not completely independent (as they are under baroclinic conditions). Under such stratification, the full $3 \mathrm{D}$ primitive equations in isentropic coordinates reduce with very little approximation to a 2D system, which obeys a conservation principle analogous to Ertel's theorem for 3D flow and has the same general form as the shallowwater equations. This model also has the advantage over the nondivergent barotropic vorticity equation and the quasigeostrophic system in that it contains an explicit representation of forcing, diabatic effects, equivalent depth, and compressibility.

Salby et al. (1990) have used this PV formulation to study stratospheric motions, but its inferred diabatic effects connected with tropospheric dynamics remain to be explored. In fact, Salby's equivalent barotropic model may be redefined in terms of a deep isentropic layer (extending from the top of the atmosphere down to some isentropic surface), so that the diabatic effects may be expressed primarily in terms of a cross-isentropic mass flux at the lower boundary of the layer. This model has the great advantage over the one-layer temperature model in Part I in that no neglect of vertical advection is required; indeed, the PV diabatic effects essentially take the form of a vertical mass flux.

In section 2 we demonstrate that the equivalent 
barotropic PV model can be derived in terms of an isentropic layer and define the adjoint model (derived in Part I) for the PV. In section 3 we evaluate the PV and discuss an adiabatic integration of the equivalent barotropic tracer model in section 4 . As a precursor to applying the adjoint method, we evaluate the diabatic terms as a residual of the model in section 5, and compare them with an estimate of the diabatic mass fluxes at the model's lower boundary. In section 6 we examine the sensitivity of the adjoint model to dissipation, horizontal resolution, and choice of the lower bounding isentrope. In section 7 the model is applied to six European January PV anomalies, which correspond to the tropospheric temperature anomalies examined in Part I. We conclude with a discussion in section 8 .

\section{Theory}

\section{a. The equivalent barotropic potential vorticity}

The potential vorticity equation is derived here in the equivalent barotropic framework. The derivation is based on that of Salby (1989), except that a deep isentropic layer $\left(\theta_{\infty}-\theta_{0}\right)$ is considered, extending from the top of the atmosphere down to a given isentrope $\theta_{0}$, as opposed to the deep material layer considered by Salby. Thus, in this case $\theta_{0}$ is constant and does not vary with time even in the presence of diabatic effects. This modification is only relevant in the presence of diabatic effects, which it considerably simplifies.

The primitive equations in isentropic coordinates may be written as

$$
\begin{gathered}
\frac{d_{\theta} \mathbf{v}}{d t}+\dot{\theta} \frac{\partial \mathbf{v}}{\partial \theta}+f \mathbf{k} \times \mathbf{v}=-\nabla_{\theta} \Psi+R \\
\frac{d_{\theta}}{d t}\left(\frac{\partial p}{\partial \theta}\right)+\frac{\partial}{\partial \theta}\left(\dot{\theta} \frac{\partial p}{\partial \theta}\right)+\left(\frac{\partial p}{\partial \theta}\right) \nabla_{\theta} \cdot \mathbf{v}=0 \\
\frac{\partial \Psi}{\partial \theta}=c_{p} \frac{T}{\theta},
\end{gathered}
$$

where $\Psi$ is the Montgomery potential, $R$ is friction, $c_{p}$ is the specific heat capacity at constant pressure, and the subscript $\theta$ denotes "at constant potential temperature." Taking $\mathbf{k} \cdot \nabla \times(1)$ yields the vorticity equation

$$
\begin{aligned}
\frac{d_{\theta}}{d t}(\zeta+f) & =-(\zeta+f) \nabla_{\theta} \cdot \mathbf{v} \\
& -\frac{\partial}{\partial x}\left(\dot{\theta} \frac{\partial v}{\partial \theta}-R_{y}\right)+\frac{\partial}{\partial y}\left(\dot{\theta} \frac{\partial u}{\partial \theta}-R_{x}\right),
\end{aligned}
$$

where $R_{x}=\partial R / \partial x$ and $R_{y}=\partial R / \partial y$. We now make the substitution

$$
\begin{aligned}
& \mathbf{v}=A(\theta)\langle\mathbf{v}\rangle, \\
& \dot{\theta}=B(\theta) \dot{\theta}_{0},
\end{aligned}
$$

such that

$$
\langle\mathbf{v}\rangle=\frac{1}{p_{0}} \int_{\infty}^{\theta_{0}} \mathbf{v}\left(\frac{\partial p}{\partial \theta}\right) d \theta
$$

is the vertical average of $\mathrm{v}$ above the isentrope $\theta_{0}$, and where $A(\theta)$ and $B(\theta)$ define the vertical structures of $\mathrm{v}$ and $\dot{\theta}$, respectively, such that $\langle A\rangle=1$ and $p_{0}=p\left(\theta_{0}\right)$. The vorticity equation then yields

$$
\begin{aligned}
& \left(\frac{\partial}{\partial t}+A(\theta)\langle\mathbf{v}\rangle \cdot \nabla\right)\left(\langle\zeta\rangle+\frac{f}{A(\theta)}\right) \\
& =-A(\theta)\left(\langle\zeta\rangle+\frac{f}{A(\theta)}\right) \nabla \cdot\langle\mathbf{v}\rangle \\
& \quad-\frac{\partial}{\partial x}\left(B(\theta) \dot{\theta}_{0}\langle v\rangle \frac{\partial}{\partial \theta} \ln A-\frac{R_{y}}{A(\theta)}\right) \\
& \quad+\frac{\partial}{\partial y}\left(B(\theta) \dot{\theta}_{0}\langle u\rangle \frac{\partial}{\partial \theta} \ln A-\frac{R_{x}}{A(\theta)}\right)
\end{aligned}
$$

Since both $A(\theta)$ and $B(\theta)$ are functions of $\theta$ alone, we may write

$$
\left\langle B(\theta) \frac{\partial}{\partial \theta} \ln A\right\rangle=\frac{1}{\theta_{00}}
$$

where $\theta_{00}$ is a reference value related to the equivalent depth. Both profiles are arbitrary, but not independent of each other. Writing $R=A(\theta)\langle R\rangle$ and taking \langle\rangle of $(6)$ then yields

$$
\begin{aligned}
\left(\frac{\partial}{\partial t}\right. & +\langle\mathbf{v}\rangle \cdot \nabla)(\langle\zeta\rangle+f)=-(\langle\zeta\rangle+f) \nabla \cdot\langle\mathbf{v}\rangle \\
& -\frac{\partial}{\partial x}\left(\langle v\rangle \frac{\dot{\theta}_{0}}{\theta_{00}}-\left\langle R_{y}\right\rangle\right)+\frac{\partial}{\partial y}\left(\langle u\rangle \frac{\dot{\theta}_{0}}{\theta_{00}}-\left\langle R_{x}\right\rangle\right) .
\end{aligned}
$$

Defining $p_{0} Q \equiv\langle\zeta\rangle+f$, where $p_{0}$ is the pressure at $\theta_{0}$, then yields the conservation form:

$$
\begin{aligned}
\frac{\partial}{\partial t}\left(p_{0} Q\right) & +\frac{\partial}{\partial x}\left(\langle u\rangle p_{0} Q+\langle v\rangle \frac{\dot{\theta}_{0}}{\theta_{00}}-\left\langle R_{y}\right\rangle\right) \\
+ & \frac{\partial}{\partial y}\left(\langle v\rangle p_{0} Q-\langle u\rangle \frac{\dot{\theta}_{0}}{\theta_{00}}+\left\langle R_{x}\right\rangle\right)=0 .
\end{aligned}
$$

This is precisely the theorem discussed by Haynes and McIntyre (1987, 1990), here in the context of the equivalent barotropic model. Because of the conservation form of (7), the volume integral of $Q$ over the layer is constant, even in the presence of diabatic heating and friction. As noted by Haynes and McIntyre, $p_{0}(x, y)$ plays the role of a density, so that $p_{0} Q$ is effectively the column-integrated amount of tracer per unit area $(S)$. Thus,

$$
\frac{\partial}{\partial t} \int_{S} \int_{p} Q d p d S=\frac{\partial}{\partial t} \int_{S} p_{0} Q d S=-\int_{S} \nabla \cdot \mathbf{J} d S=0,
$$


from (7) where $\mathbf{J}=\left(\langle u\rangle p_{0} Q+\langle v\rangle \dot{\theta}_{0} / \theta_{00}-\left\langle R_{y}\right\rangle\right) \mathbf{i}$ $+\left(\langle v\rangle p_{0} Q-\langle u\rangle \dot{\theta}_{0} / \theta_{00}+\left\langle R_{x}\right\rangle\right) \mathbf{j}$, assuming periodic boundary conditions.

To derive the PV equation from (7), we require the continuity equation for the equivalent barotropic model. Substituting for $\mathbf{v}$ in (2) using (5) yields

$$
\begin{aligned}
\left(\frac{\partial}{\partial t}+A(\theta)\langle\mathbf{v}\rangle \cdot \nabla\right)\left(\frac{\partial p}{\partial \theta}\right)+ & \frac{\partial}{\partial \theta}\left(\dot{\theta} \frac{\partial p}{\partial \theta}\right) \\
& +A(\theta)\left(\frac{\partial p}{\partial \theta}\right) \nabla_{\theta} \cdot\langle\mathbf{v}\rangle=0 .
\end{aligned}
$$

Integrating over the isentropic layer and recalling that $\langle A\rangle=1$ then gives

$$
\left(\frac{\partial}{\partial t}+\langle\mathbf{v}\rangle \cdot \nabla\right) p_{0}+p_{0} \nabla \cdot\langle\mathbf{v}\rangle+\left(\dot{\theta} \frac{\partial p}{\partial \theta}\right)_{0}=0,
$$

where the subscript zero again denotes that the term relates to the lower bounding isentrope, $\theta_{0}$. Thus, the pressure of this isentrope depends only on adiabatic transport and the diabatic mass flux across it.

Subtracting $Q \times(8)$ from (7) finally yields the PV equation

$$
\left(\frac{\partial}{\partial t}+\langle\mathbf{v}\rangle \cdot \nabla\right) Q=F_{Q}
$$

where

$$
Q=\frac{(\langle\zeta\rangle+f)}{p_{0}}
$$

is the equivalent barotropic $\mathrm{PV}$, and

$$
\begin{aligned}
F_{Q}=\frac{1}{p_{0}}\left\{Q\left(\dot{\theta} \frac{\partial p}{\partial \theta}\right)_{0}\right. & -\frac{\partial}{\partial x}\left(\langle v\rangle \dot{\theta}_{0} / \theta_{00}-\left\langle R_{y}\right\rangle\right) \\
+ & \left.\frac{\partial}{\partial y}\left(\langle u\rangle \dot{\theta}_{0} / \theta_{00}-\left\langle R_{x}\right\rangle\right)\right\}
\end{aligned}
$$

are the diabatic effects. As usual, the twisting terms are small by quasigeostrophic scaling, so that the effect of diabatic heating on the local, equivalent barotropic potential vorticity $Q$ is primarily associated with a crossisentropic flux of mass across $\theta_{0}$. Here $\dot{\theta}_{0}$ represents a "vertical" velocity and $\partial p / \partial \theta$ is a measure of the mass in an isentropic layer.

The mass flux has a "concentrating" or "diluting" effect on the PV "substance" $\left(p_{0} Q\right)$ within the equivalent barotropic isentropic layer so that local changes are possible while the layer integral remains constant. For example, in the case of a heating $\left(\dot{\theta}_{0}>0\right)$, with stable stratification $(\partial p / \partial \theta)_{0}<0$, the isentrope $\theta_{0}$ sinks to higher pressure so that there is effectively a mass flux into the equivalent barotropic isentropic layer diluting $Q$ consistent with $F_{Q}<0$ in (9c). However, the layer has become locally thicker so that a layer integral of the PV substance remains unchanged.

\section{$b$. The adjoint model}

The tracer equation corresponding to $(9 a)$ reads

$$
\left(\frac{\partial}{\partial t}+\langle\mathbf{v}\rangle \cdot \nabla\right) Q+\kappa \nabla^{4} Q=F_{Q},
$$

where a scale-selective diffusion is added to facilitate numerical integration, and $\langle\mathbf{v}\rangle$ is a prescribed wind. The adjoint of (10), as described in Part $I$, is then

$$
-\left(\frac{\partial}{\partial t}+\langle\mathbf{v}\rangle \cdot \nabla\right) Q^{*}+\kappa \nabla^{4} Q^{*}=F^{*}
$$

where $Q^{*}$ is the adjoint variable and $F^{*}$ is a local conjugate-forcing step function (to be specified) that is nonzero only in the region $G$ and time interval $I$, where $|I|=\Delta t=t_{1}-t_{0}$. Following Marchuk (1974), (10) and (11) may then be combined (via the extended Green's identity), choosing $Q^{*}\left(t=t_{1}\right)=0$, to describe a regional $P V$ anomaly $\bar{Q}^{\prime} G, I$ defined with respect to reference value $Q_{b}$, as described in Part I:

$$
{\overline{Q^{\prime}}}^{G, I}=\int_{H}\left(Q_{0}-Q_{b}\right) Q_{0}^{*} d a+\int_{H} \int_{t_{0}}^{t_{1}} Q^{*} F_{Q} d t d a
$$

where $H$ denotes integration over the hemisphere and where $Q_{0}$ denotes $Q\left(t=t_{0}\right)$, etc. The first term on the rhs is referred to in the following as the "initial conditions (ICs) term," and the double integral as the "forcing effects," or simply "forcing term." Comparing (11) with its temperature counterpart in Part I, it is clear that the information tracer $Q^{*}$ only differs from $T^{*}$ through the differing vertical averages used to define the advecting wind in the one-layer model. The hemispheric spectral model developed in Part $I$ is used to numerically evaluate (12) by integrating (11) in sections 6 and 7 below.

\section{Evaluation of the potential vorticity}

The vertical averages $\langle\mathbf{v}\rangle$ and $\langle\zeta\rangle$ defined by (5) were computed by first evaluating the pressure of a chosen isentrope, $p_{0}(x, y)$ interpolating $\theta$ linearly between pressure surfaces. Approximate averages by pressure above the isentropic surface were then computed, using streamfunction data at 100,200,300,400, 500,700 , and $850 \mathrm{mb}$, from the same six-January dataset (0000 UTC daily, 1980-85) used in Part I from the European Centre for Medium-Range Weather Forecasts.

The $310 \mathrm{~K}$ surface was chosen to be the lower bounding isentrope $\left(\theta_{0}\right)$ defining the equivalent barotropic model. The pressure-height of this surface averaged over the six Januarys is shown in Fig. 1, along 

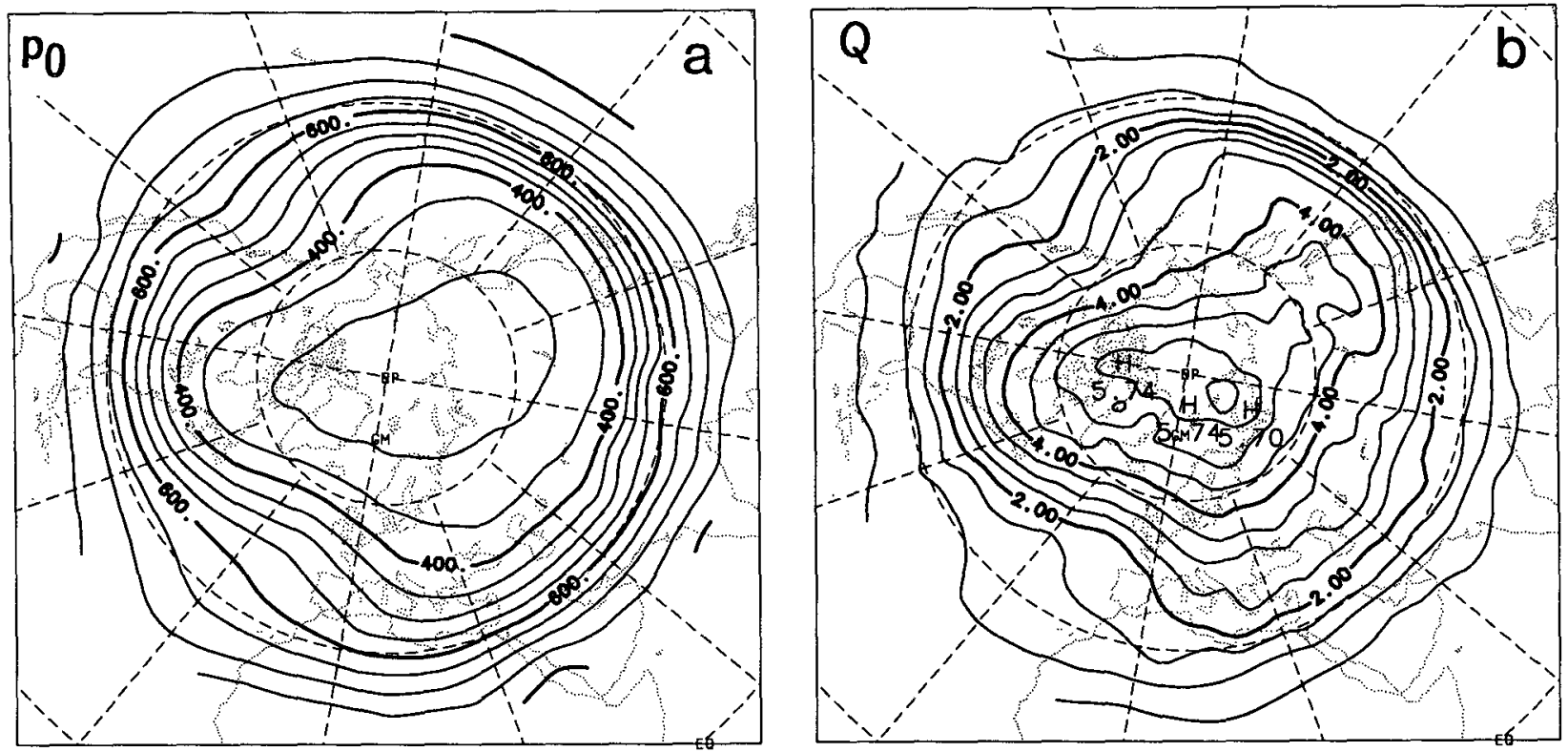

FIG. 1. Six-January T42 climatologies of (a) $p\left(\theta=310 \mathrm{~K}\right.$ ) (contour interval $50 \mathrm{mb}$ ), (b) $Q$ for $p_{0}=310 \mathrm{~K}$ [contour interval $0.5 \mathrm{QU}$; $\left.1 \mathrm{QU} \equiv 1 \times 10^{-5} \mathrm{~s}^{-1}(100 \mathrm{mb})^{-1}\right]$. All maps are polar stereographic projections, with Greenwich meridian bottom-left center.

with the associated $Q$ distribution, both truncated triangularly at total wavenumber $n=42$ (T42). The 310 $\mathrm{K}$ surface extends in the mean from near the tropopause in the polar region to about $800 \mathrm{mb}$ at $20^{\circ} \mathrm{N}$ and has quite a smooth distribution in the six-monthly mean. In contrast, the equivalent barotropic potential vorticity has a "crinkly" structure at T42 even in the six-January mean, though it closely mirrors $p\left(\theta_{0}\right)$ at T21. These-presumably spurious-wiggles in $Q$ are associated with the relative vorticity and give the timemean residual forcing $F_{Q}$ a noisy small-scale signature at $\mathrm{T} 42$, as will be seen in section 5 . The magnitude of $Q$ ranges from a maximum of about 5 QU ( 1 QU ( $Q$ unit $\left.) \equiv 1.0 \times 10^{-5} \mathrm{~s}^{-1}(100 \mathrm{mb})^{-1}\right)$ near the pole, to about $1-2 \mathrm{QU}$ at $30^{\circ} \mathrm{N}$.

\section{Forward integration of the equivalent barotropic tracer model}

An idea of the importance of the forcing $F_{Q}$ in (10) may be gained by integrating (10) with $F_{Q}=0$ and $\langle\mathbf{v}\rangle$ prescribed from observations. Figure 2 compares the simulated $Q$ with observations after 7 and 28 days, starting from the observed $Q$ at 0000 UTC 1 January 1985 , truncating at T21 with dissipation time scale $t_{D}$ $=1$ day at $n=21$. A $1 / 2$-hour time step is used in all experiments, interpolating the daily observations linearly in time.

The ability of the one-layer tracer model to simulate the evolution of $Q$ is distinctly better than in the case of tropospheric temperature in Part I ( the $T$ case hereafter) and is quite reasonable at day 7 . The equivalent barotropic model appears to conserve $Q$ better locally than the tropospheric model does $T$, in spite of $Q$ being a more "difficult" derivative quantity than temperature. Amplitudes are maintained somewhat better than in the $T$ case, though again small-wavelength features lack amplitude. In particular, incursions of low- $Q$ air into the vortex are underestimated by the simulation, a feature with severe dynamical implications for the evolution of a real flow (i.e., if the wind were not constantly prescribed). After 28 days, the meridional PV gradient is considerably weakened at high latitudes with polar values reduced, consistent with zonal mixing (and dissipation ) in the absence of diabatic forcing. The dissipation represents the small-scale mixing of $Q$ that is characteristic of any flow evolution and is thus crucial to the success of the tracer-model integration.

As found in the stratosphere (McIntyre and Palmer 1984), the observed $Q$-field evolution appears to be characterized by mixing with incursions of low- $Q$ air into the vortex, rather than vice versa. Compared to the corresponding $T$ fields, the observed $Q$ fields present a more distorted vortex with smaller-scale larger-amplitude intrusion features. We have repeated the above experiment at $\mathrm{T} 42$ resolution (with correspondingly much weaker dissipation), but the higher spatial resolution brings only slight improvement in the simulation, indicating the importance of $F_{Q}$ for these features. We note, however, that at $\mathrm{T} 42$, the observed $Q$ fields have a much more small-scale structure than their tropospheric temperature counterparts.

\section{Evaluation of the diabatic terms as a residual}

The five-January (1981-85) time-mean forcing $\hat{F}_{Q}$ (where the hat denotes the five-January mean from 

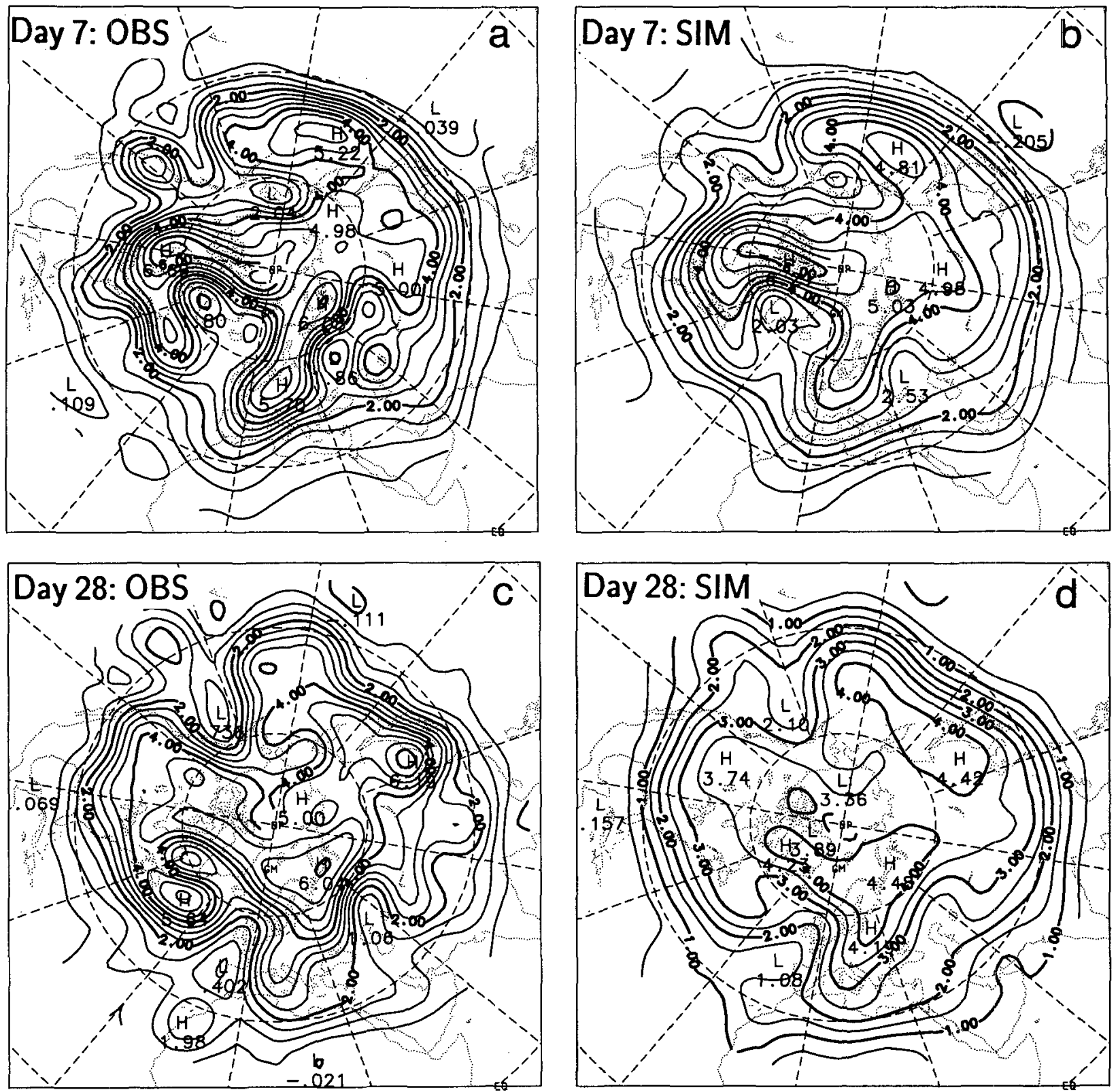

FIG. 2. Direct unforced integration of (10) at T21 with $t_{D}=1$ day at $n=21$, starting from observed $Q$ at 0000 UTC 1 January 1985 . Day 7: (a) observed $Q$, (b) simulation. Day 28: (c) observed $Q$, (d) simulation. Contour interval is 0.5 QU.

now on) derived as a residual from (10) is illustrated in Fig. 3a [ computed at T21 with the (small) diffusion contribution omitted]. January 1980 is excluded because the $\langle\zeta\rangle$ and $F_{Q}$ fields for that month exhibit a (presumably) spurious structure in the vicinity of the Tibetan Plateau. The $\hat{F}_{Q}$ field has coherent structures over east Asia and the Pacific, with the Atlantic sector being dominated by weak amplitude features. The field is rather noisy and becomes dominated by noise if the computation is performed at T42; nevertheless, the T21 component of the latter closely resembles Fig. 3a.
If the effects of dissipation and tendencies may be neglected in the five-January mean, (10) yields

$$
\hat{F}_{Q} \approx\langle\hat{\mathbf{v}}\rangle \cdot \nabla \hat{Q}+\widehat{\langle\mathbf{v}\rangle^{t} \cdot \nabla Q^{t}}
$$

where $Q^{t}=Q-\hat{Q}$, etc. Computing $\hat{F}_{Q}$ via (13) yields a very similar picture to Fig. $3 a$. It is found that it is the stationary wave component that dominates the large-scale structures seen in Fig. 3a over the Pacific and east Asia, while there is considerable cancellation with the transient term over the Atlantic and Europe. 

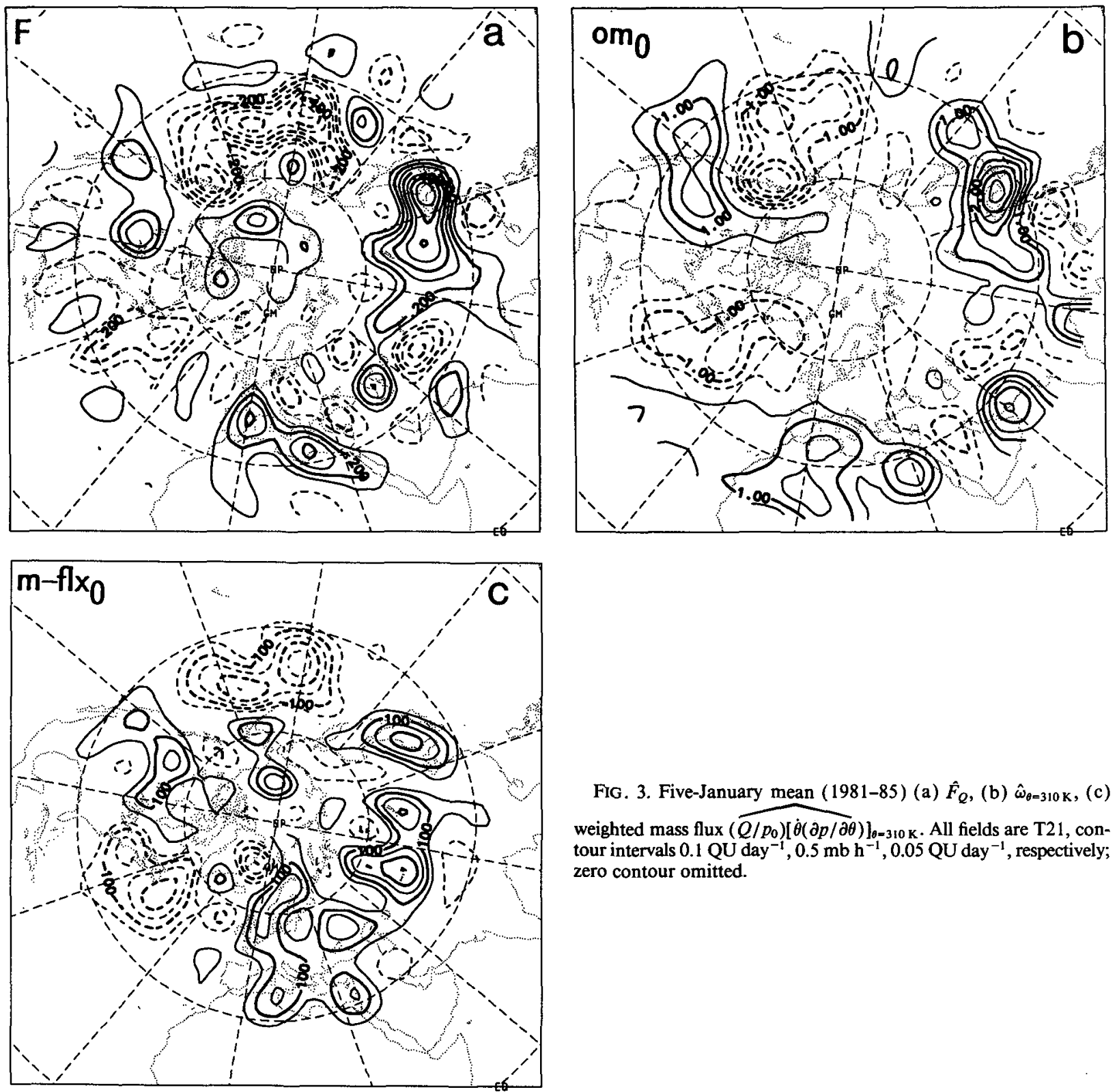

FIG. 3. Five-January mean (1981-85) (a) $\hat{F}_{Q}$, (b) $\hat{\omega}_{\theta=310 \mathrm{~K}}$, (c) weighted mass flux $\left.\left(\widehat{\left.Q / p_{0}\right)[\dot{\theta}(\partial p / \partial \theta}\right)\right]_{\theta=310 \mathrm{~K}}$. All fields are T21, contour intervals $0.1 \mathrm{QU}^{\text {day }}{ }^{-1}, 0.5 \mathrm{mb} \mathrm{h}^{-1}, 0.05 \mathrm{QU}^{\text {day }}{ }^{-1}$, respectively; zero contour omitted.

It is clear that the small-scale structure in $\hat{F}_{Q}$, at least at $\mathrm{T} 42$, is to a large extent a consequence of the (presumably spurious) wiggles in $\hat{Q}$ seen in Fig. 1b; the largest gradients being on the smallest scales. The crinkly nature of instantaneous $Q$ at high resolution is a fundamental dynamical property of $P V$ associated with the cascade to smaller scales (e.g., Juckes and McIntyre 1987), but one would hardly expect to see it in the time average.

To within the equivalent barotropic approximation, $F_{Q}$ is purely diabatic and dominated by the cross-isentropic mass flux at $\theta_{0}(9 \mathrm{c})$. A first indication of this mass flux can be gained from the vertical velocity field at $\theta_{0}$ (i.e., $\omega_{0}$ ), whose five-January mean is illustrated

in Fig. 3b. To the extent that vertical motion accompanies diabatic heating/cooling, ascent at the layer boundary tends to inject mass into the equivalent barotropic isentropic layer above, resulting in a dilution of the PV and a corresponding (diabatic) decrease in $Q$. Indeed, there is quite a reasonable correspondence between $\hat{F}_{Q}$ in Fig. 3a and $\hat{\omega}_{0}$ in Fig. 3b, although $\hat{F}_{Q}$ is somewhat noisier than $\hat{\omega}_{0}$.

In general, the relationship between diabatic heating and vertical motion is more complex, and expanding omega in isentropic coordinates we have

$$
\omega=\frac{d p}{d t}=\frac{\partial p}{\partial t}+\mathbf{v}_{\theta} \cdot \nabla_{\theta} p+\dot{\theta} \frac{\partial p}{\partial \theta}
$$


so that at $\theta=\theta_{0}$ we can express the cross-isentropic mass flux as

$$
\left(\dot{\theta} \frac{\partial p}{\partial \theta}\right)_{0}=\omega_{0}-\frac{\partial p_{0}}{\partial t}-\mathbf{v}_{0} \cdot \nabla_{\theta} p_{0}
$$

In the adiabatic case where ascent $\left(\omega_{0}<0\right)$ is not accompanied by diabatic heating $\left(\dot{\theta}_{0}=0\right)$, and in the absence of isentropic advection $\left(v_{0} \cdot \nabla_{\theta} p_{0}=0\right)$, the isentrope $\theta_{0}$ will ascend to lower pressure. However, vortex compression above $\theta_{0}$ will exactly nullify the decrease in $p_{0}$, so that $Q$ remains unchanged. On the other hand, the presence of $\dot{\theta}_{0}>0$ will be accompanied by a diabatic descent of $\theta_{0}$ and a decrease in $Q$.

Equation (14) enables an estimate of the cross-isentropic mass flux to be made, although there is often a large cancellation between $\hat{\omega}_{0}$ and $\widehat{v_{0} \cdot \nabla_{\theta} p_{0}}$ (i.e., where vertical motion is largely adiabatic), so that the residual diabatic effects may be unreliable. Figure $3 \mathrm{c}$ illustrates the weighted mass flux $\left.\left(\widehat{\left.Q / p_{0}\right)[\dot{\theta}(\partial p / \partial \theta}\right)\right]_{0}$, which provides the main diabatic term in the PV equation (9) in the five-January mean. The general picture is one of diluting (i.e., "upward") mass fluxes over the oceans and concentrating mass flux (i.e., "downward" out of the layer) over the continents. Indeed, this pattern is in rough agreement with the tropospheric diabatic heating distribution discussed in Part I, with heating over the oceans and cooling over the continents.

Over the western hemisphere the weighted mass flux generally accounts for $\hat{F}_{Q}$ quite well and somewhat better than does $\hat{\omega}_{0}$, while the comparison is not as good over Eurasia. The agreement is best on larger scales and the comparison improves if the fields are spectrally smoothed to T14. The weighted mass flux in Fig. $3 \mathrm{c}$ accounts for the order of magnitude of $\hat{F}_{Q}$, although the amplitude of the latter is somewhat larger (note differing contour intervals in Figs. 3a and 3c).

\section{Application of the adjoint method: Sensitivity experiments}

The adjoint method is now applied to the same central European target region $G$ considered in Part I, using the local $F^{*}$ function with amplitude $F^{*}=1$ IDU day $^{-1}$ inside $G$ (and zero elsewhere), where IDU are arbitrary "information density units" with dimensions of (area) ${ }^{-1}$. Again we take January 1985 and integrate the adjoint equation (11) backward from $t_{1}=2400$ UTC 31 January, with $Q_{1}^{*}=0$, to $t_{0}=0000$ UTC 1 January. Equation (12) is then evaluated using the same finite-difference scheme as in Part I. Before attempting to interpret the regional PV in terms of its contributions from the initial conditions (ICs) and forcing terms in (12), we first investigate the sensitivity of the results to dissipation, horizontal resolution, and choice of lower-bounding isentrope $\theta_{0}$.

\section{a. Dissipation and horizontal resolution}

Table 1 illustrates the terms in (12) for various dissipation strengths, using a horizontal truncation at $\mathrm{T} 21$. We set the reference value $Q_{b}=0$ in (12), so that $\bar{Q}^{\prime}, I$ is the total averaged PV within the target domain $(G, I)$. The units in all the following tables are scaled to be $1 / 10$ QU. Equation (12) is generally satisfied to well within one-tenth of the forcing-integral magnitude in all the following experiments, with smaller error at $\mathrm{T} 21$ than at T42.

The average PV over central Europe during January 1985 is supplied almost exclusively by the initial-conditions term for small dissipation. This suggests that the forcing integral is largely an artifact of the dissipation, and any physical forcing effects are smothered; the forcing term merely compensating for information lost to dissipation in the adjoint integration. Moreover, any amount of dissipation appears to be too much, and the forcing integral is sensitive to $t_{D}$ even at weak dissipation. Unfortunately, decreasing the dissipation strength in Table 1 also leads to the increasing occurrence of locally negative tracer values at time $t=t_{0}$, as indicated in the far-right column in Table 1. Such negative information values are an artifact of the numerics so that at T21 resolution there is clearly a tradeoff between dissipating information and generating negative information tracer values.

One way to improve on this compromise is to resolve the information tracer better, as in Table 2, which shows the sensitivity of the adjoint problem to dissipation at $\mathrm{T} 42$ resolution.

In the first row of Table 2, the dissipation strength is equal to $t_{D}=1$ day at $n=21$, and both ICs and

TABLE 1. Values of terms in (12) for January 1985 in $1 / 10$ QU with $Q_{b}=0$, as a function of dissipation time scale $t_{D}$ at $n=21$, using T21 horizontal truncation. The far-right column gives the range of local magnitudes of $Q_{0}^{*}$ in (arbitrary) information density units (IDU).

\begin{tabular}{ccccc}
\hline \hline $\begin{array}{c}\text { Dissipation } \\
\text { (days) }\end{array}$ & $\begin{array}{c}\text { Anomaly } \\
\overline{\bar{Q}^{\prime G, I}}\end{array}$ & $\begin{array}{c}\mathrm{ICs} \\
\int_{H}\left(Q_{0}-Q_{b}\right) Q_{0}^{*}\end{array}$ & $\begin{array}{c}\text { Forcing } \\
\int_{H} \int_{I} Q^{*} F_{Q}\end{array}$ & $\begin{array}{c}\text { Tracer magnitude } Q_{0}^{*} \\
\text { (IDU) }\end{array}$ \\
\hline $1 / 4$ & 40.43 & 35.31 & 5.17 & -0 to 10 \\
1 & & 38.05 & 2.39 & -1 to 13 \\
4 & & 39.46 & 0.99 & -4 to 15 \\
16 & 40.24 & 0.19 & -9 to 16 \\
$\infty$ & & 40.71 & -0.27 & -11 to 17 \\
\hline
\end{tabular}


TABLE 2. Values of terms in (12) for January 1985 in $1 / 10$ QU with $Q_{b}=0$, as a function of dissipation time scale $t_{D}$ at $n=42$, using T42 horizontal truncation. As Table 1.

\begin{tabular}{ccccc}
\hline \hline $\begin{array}{c}\text { Dissipation } t_{D} \\
\text { (days) }\end{array}$ & $\begin{array}{c}\text { Anomaly } \\
\overline{Q^{G}, I}\end{array}$ & $\begin{array}{c}\text { ICs } \\
\int_{H}\left(Q_{0}-Q_{b}\right) Q_{0}^{*}\end{array}$ & $\begin{array}{c}\text { Forcing } \\
\int_{H} \int_{I} Q^{*} F_{Q}\end{array}$ & $\begin{array}{c}\text { Tracer magnitude } Q_{0}^{*} \\
\text { (IDU) }\end{array}$ \\
\hline 0.07 & 40.58 & 37.94 & 2.62 & -0 to 13 \\
1 & & 40.04 & 0.63 & -2 to 25 \\
4 & & 40.02 & 0.67 & -6 to 27 \\
16 & 39.79 & 0.72 & -11 to 26 \\
$\infty$ & & 39.62 & 0.76 & -14 to 26 \\
\hline
\end{tabular}

forcing integrals compare well with their $\mathrm{T} 21 / t_{D}=1$ day counterparts in Table 1 , indicating robustness. In contrast to Table 1 , the magnitude of the forcing integral does reach a minimum for finite dissipation $\left(t_{D}\right.$ $=1$ day at $n=42$ ), for which negligible negative tracer values occur in the initial conditions. At weaker dissipations, the forcing integral increases only slightly, despite the increasingly noisy nature of the initial conditions, indicating massive spatial cancellation between regions of positive and negative $Q_{0}^{*}$ at zero dissipation.

It appears that sufficiently high horizontal resolution is essential when applying the adjoint method to the $\mathrm{PV}$, presumably because of the size of the advection velocities associated with the equivalent barotropic model. In addition, moderately strong dissipation is required, if the $Q_{0}^{*}$ field is not to become meaningless. A T42 truncation with $t_{D}=1$ day at $n=42$ does appear to be satisfactory, although there is still no guarantee that dissipation is not contaminating the result.

\section{b. Experiments with different layer depths}

The effect of different choices of the isentrope bounding the equivalent barotropic layer from below $\left(\theta_{0}\right)$ is illustrated in Table 3 , where T 21 resolution with $t_{D}=1$ day at $n=21$ is used.

The ratio of the magnitudes of the forcing-to-ICs integrals is almost constant for $\theta_{0} \geqslant 310 \mathrm{~K}$, while for the two lowest isentropes (which intersect the ground surface and do not intersect the stratosphere in the mean ) the ratio is smaller and even changes sign. Thus the importance of the diabatic effects remains fairly constant for $\theta_{0} \geqslant 310 \mathrm{~K}$, but decreases for lower $\theta_{0}$, perhaps associated with decreased static stability, or intersection of $\theta_{0}$ with the ground. Consequently, there appears to be no advantage in choosing a more elevated $\theta_{0}$ than $310 \mathrm{~K}$, while lower isentropes have the problem of intersection with the ground.

\section{A comparison of six Januarys over central Europe}

We are now in a position to apply the adjoint method to central European PV anomalies for the six Januarys of our dataset, and to compare the results with those obtained using tropospheric temperature for the same situations in Part I. Table 4 presents the magnitudes of the integrals in (12) for $Q$ for all six Januarys, in terms of the six-January grand mean and deviations therefrom. The experiments have been carried out at T42 resolution with $t_{D}=1$ day at $n=42$, though rather similar results are obtained at $\mathrm{T} 21$ with $t_{D}=1$ day at $n=21$.

In contrast to the temperature case in Part I, the diabatic effects on the central European target region are almost zero in the six-January mean. This suggests that $Q$ is more conservative than tropospheric temperature was in Part I, consistent with the better forward integration in section 4. Since $Q_{b}=0$, the ICs describe the advection of climatological PV, which may be removed to give zero ICs by setting $Q_{b}=3.4$ QU. Figure 4 shows the spatial fields associated with the six-January mean ICs, with $Q_{b}=3.4 \mathrm{QU}$. The $Q_{0}^{*}$ field is superimposed in bold on the $\left(Q_{0}-Q_{b}\right)$ field in Fig. $4 \mathrm{a}$, illustrating a rather well-mixed $Q_{0}^{*}$ distribution, with a maximum over the target region. The information tracer is more rapidly advected than in the climato-

TABLE 3. Values of integrals in (12) for January 1985 in $1 / 10$ QU for various values of $\theta_{0}$. As in Table 1 for $t_{D}=1$ day. The far right-hand column gives the ratio of forcing to ICs integrals, multiplied by 100 .

\begin{tabular}{ccccc}
\hline & Anomaly & ICs & Forcing & \\
$\theta_{0}(\mathrm{~K})$ & $\bar{Q}^{\prime G, I}$ & $\int_{H}\left(Q_{0}-Q_{b}\right) Q_{0}^{*}$ & $\int_{H} \int_{I} Q^{*} F_{Q}$ & $($ Forc $/ \mathrm{ICs}) \times 100$ \\
\hline 290 & 21.76 & 22.05 & -0.26 & -1.2 \\
300 & 29.97 & 29.61 & 0.36 & 1.2 \\
310 & 40.43 & 38.05 & 2.39 & 6.3 \\
320 & 48.22 & 45.91 & 2.35 & 6.1 \\
330 & 55.35 & 52.26 & 3.31 & 6.3 \\
\hline
\end{tabular}


TABLE 4. Values of integrals in (12) in $1 / 10 \mathrm{QU}$ for six-January mean and deviations therefrom. T42, $t_{D}=1$ day $/ n=42, Q_{b}=0$. The far-right column contains the ratio of deviations between forcing and ICs.

\begin{tabular}{ccccc}
\hline \hline & Anomaly & ICs & \\
January & $\int_{H}\left(Q_{0}-Q_{b}\right) Q_{0}^{*}$ & $\int_{H} \int_{t_{0}}^{t_{1}} Q^{*} F_{Q}$ & Forcing/ICs \\
\hline & Mean & \\
& +34.26 & +34.64 & -0.45 \\
& Deviations & & \\
1980 & +2.63 & +0.77 & +1.77 & +2.29 \\
1981 & +1.61 & +2.74 & -0.95 & -0.35 \\
1982 & -2.37 & -0.73 & -2.09 & +2.86 \\
1983 & -7.23 & -5.35 & -1.81 & +0.34 \\
1984 & -0.96 & +5.83 & +2.00 & -0.71 \\
1985 & +6.32 & +5.08 & +0.20 \\
\hline
\end{tabular}

logical tropospheric $T$ case in Part I. The climatological ICs (Fig. 4b) have a predominantly zonal structure with much spatial cancellation as in the $T$ case, but with a local maximum north of the target region.

Compared to the corresponding temperature anomalies (Table 6 in Part I), it is clear that anomalously cold Januarys are associated with positive $Q$ anomalies (and vice versa), and that the strongest $Q$ anomalies (1983 and 1985) are associated with the strongest tropospheric temperature anomalies. This suggests that the layer depth plays a major role in determining $Q$. Hoskins et al. (1985) have found that anomalies in static stability and vorticity contribute roughly equally to observed atmospheric isentropic PV anomalies, and we indeed find that anomalously cold Januarys also have cyclonic vorticity anomalies in the upper troposphere.

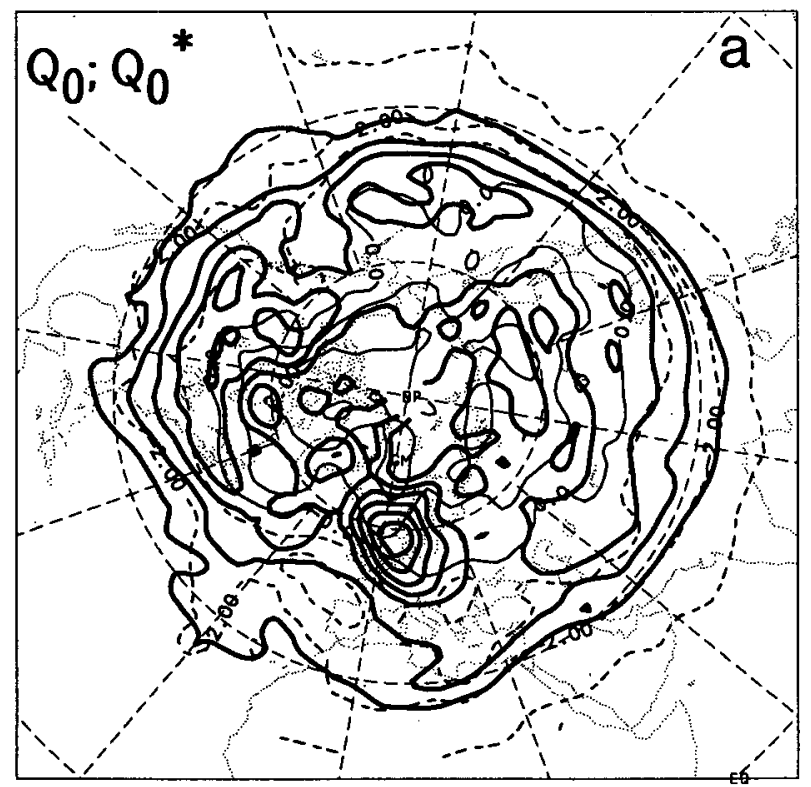

January 1983 and 1985 have the strongest PV anomalies (both $>0.5 \mathrm{QU}$ ), and as in the case of temperature these anomalies are accounted for to a large extent by anomalous ICs, especially in 1985 in the PV case. Indeed for all six anomalies, the ratio of the forcing to the IC anomalies is of the same order to that found in the $T$ case and is often rather similar. Thus, for the $Q$ anomalies, the forcing term is generally just as important as it was for the corresponding tropospheric temperature anomalies in Part I. Figure 5 illustrates the spatial fields associated with the ICs for Januarys 1983 and 1985, again in terms of the PV distribution on 1 January $\left(Q_{0}-Q_{b}\right)$ with $Q_{0}^{*}$ superimposed, and in terms of their product. In both cases, the spatial distributions are quite similar to those obtained in Part I using tropospheric temperature, with high PVs (Fig. 5d) associated with low tropospheric temperatures

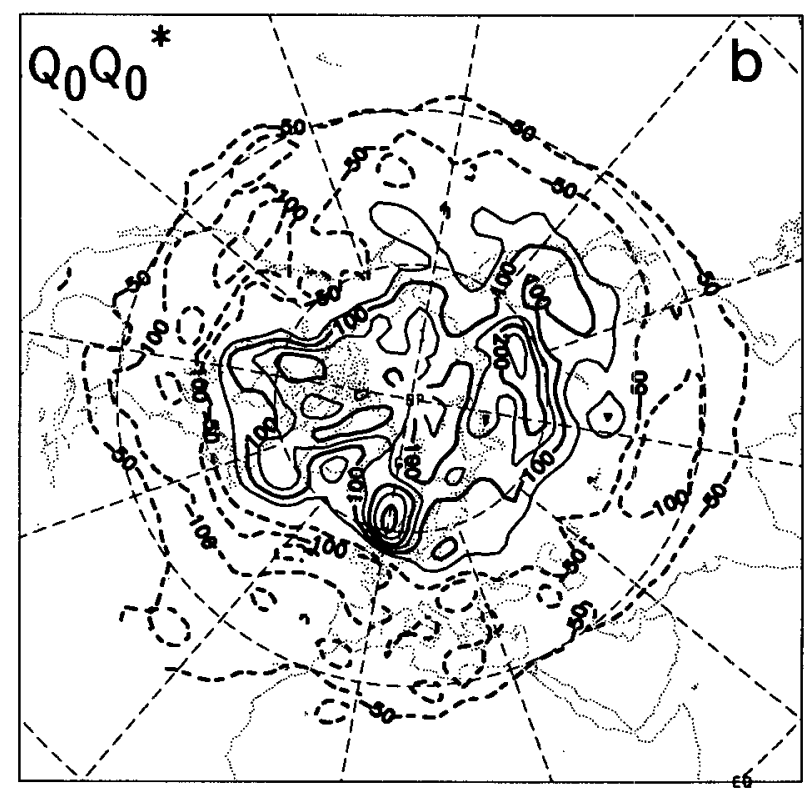

FIG. 4. Six-January mean ICs. (a) $\left(Q_{0}-Q_{b}\right)$ (light) and $Q_{0}^{*}$ (heavy, zero contour omitted), with contour intervals 1 QU and 1 IDU (information density units), respectively; (b) $\left(Q_{0}-Q_{b}\right) Q_{0}^{*} / \Delta t$, contour interval 0.05 IDU QU day ${ }^{-1}$, zero contour omitted; $Q_{b}=3.4 \mathrm{QU}$, T42 resolution. 

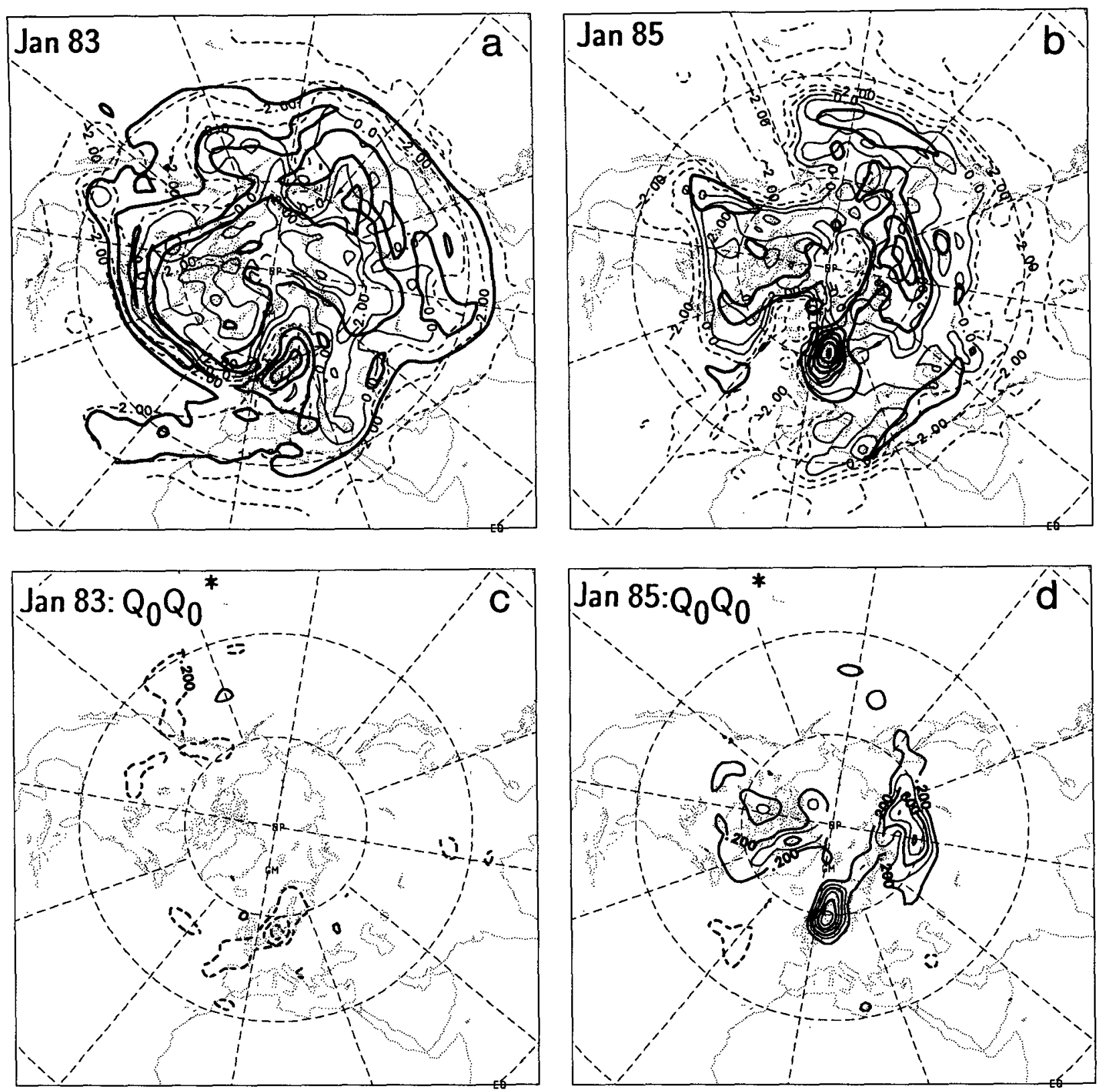

FIG. 5. Initial conditions for January 1983 (left) and 1985 (right), respectively: (a) and (b) $\left(Q_{0}-Q_{b}\right)$ (light, contour interval 1 QU) and $Q_{0}^{*}$ (heavy, contour intervals 2 and 4 IDU, respectively, zero contour omitted); (c) and (d) $\left(Q_{0}-Q_{b}\right) Q_{0}^{*} / \Delta t$, contour interval 0.2 IDU QU day ${ }^{-1}$, zero contour omitted; $Q_{b}=3.4 \mathrm{QU}, \mathrm{T} 42$ resolution.

(Fig. 6b in Part I), and vice versa (cf. Fig. 5c with Fig. $10 \mathrm{~b}$ in Part I). Again, the $Q_{0}^{*}$ field is more spread out around the hemisphere than $T_{0}^{*}$, especially in January 1983, due to the faster flow speeds characteristic of the equivalent barotropic model. These two months form a striking contrast, with the low-PV anomaly in January 1983 being a result of advection of low PV from the southwest, while the January 1985 high-PV anomaly clearly has its roots in the high-PV reservoirs to the north and northeast.

Despite large similarities with the temperature case in Part I, there is by no means a one-to-one correspondence between the respective spatial fields. There are marked high-PV sources in January 1985 over northern Europe and west Greenland without direct counterparts in the $T$ case. In January 1983 the main 

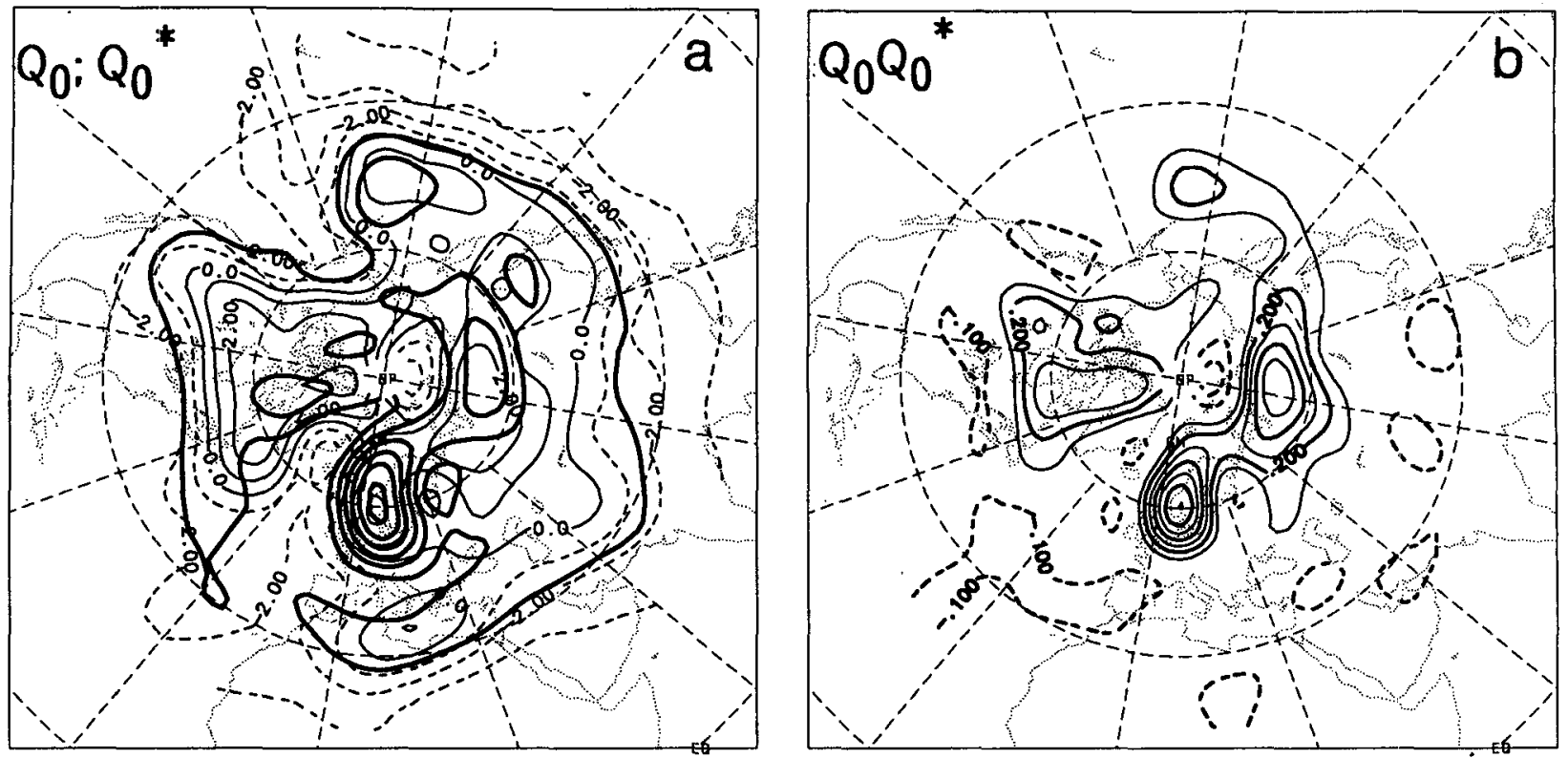

FIG. 6. January 1985 initial conditions from T21 experiment. (a) $\left(Q_{0}-Q_{b}\right)$ (light) with $Q_{0}^{*}$ (heavy, zero contour omitted), with contour intervals $1 \mathrm{QU}$ and 2 IDU, respectively; (b) $\left(Q_{0}-Q_{b}\right) Q_{0}^{*} / \Delta t$, contour interval 0.1 IDU QU day ${ }^{-1}$, zero contour omitted; $Q_{b}=3.4 \mathrm{QU}$.

low-PV sources are over the west coasts of the continents, and not over the Gulf of Mexico as in the temperature case.

Figure 6 shows the spatial field of the ICs for January 1985 carrying out all computations at T21 resolution with $t_{D}=1$ day at $n=21$. The reduced resolution yields smoother fields, but the main features are still resolved. Indeed, computing at T21 yields very similar results to the $\mathrm{T} 21$ component of the $\mathrm{T} 42$ results, for both ICs and forcing fields.

We turn now to the spatial distribution of the forcing term-that is, the diabatic effects that contribute to the PV budget over central Europe-illustrated in Fig. 7, where the distributions have been spectrally smoothed to T21. In the five-January mean of $\overline{F_{Q} Q^{*}}$ (Fig. 7a), one sees that virtually all the main features in $\hat{F}_{Q}$ (Fig. 3a) play a role in the PV budget over Europe, although Table 4 indicates that the spatial cancellation is almost complete, and the regional budget vanishes. Figures $7 \mathrm{~b}, \mathrm{c}$ show the deviations of the forcing term in January 1983 and 1985, respectively. The distributions have larger amplitudes than the mean (note larger contour interval) and are dominated by the smallest scales admitted by the $\mathrm{T} 21$ filter, and resist an interpretation in terms of weather regimes, as was possible in the temperature case. Again, the distributions are much more hemispheric than their temperature counterparts, so that remote diabatic effects apparently play a larger role in the PV budget over Europe during both months than in the temperature case. However, the pronounced spatial cancellation in the PV case suggests that remote forcing effects may be negligible when integrated spatially.

\section{Discussion}

The adjoint method has been applied to regional anomalies of equivalent barotropic PV, with the idea that this dynamic tracer might produce dynamical insight not amenable through the analysis of temperature in Part I. The equivalent barotropic model does seem to have much better conservative qualities than the one-layer tropospheric temperature model examined in Part $\mathrm{I}$, as demonstrated by a forward integration. Furthermore, the residual forcing term may be interpreted directly in terms of diabatic effects, whereas in the one-layer tropospheric temperature model considered in Part I there was a large adiabatic component associated with vertical motions. However, an analysis of this PV forcing term constructed from observations indicates that it is inherently noisy. In the five-January time mean, we were partially able to account for the forcing term's spatial distribution via an estimate of the diabatic mass flux at the isentropic base of the model. The limited success may reside with inaccuracies of the mass flux itself, whose computation as a residual involves large cancellations. Inaccuracies in the interpolated omega field and in the equivalent barotropic formulation certainly also play a role. The noisiness of the PV residual forcing increases dramatically when the horizontal resolution of the compu- 

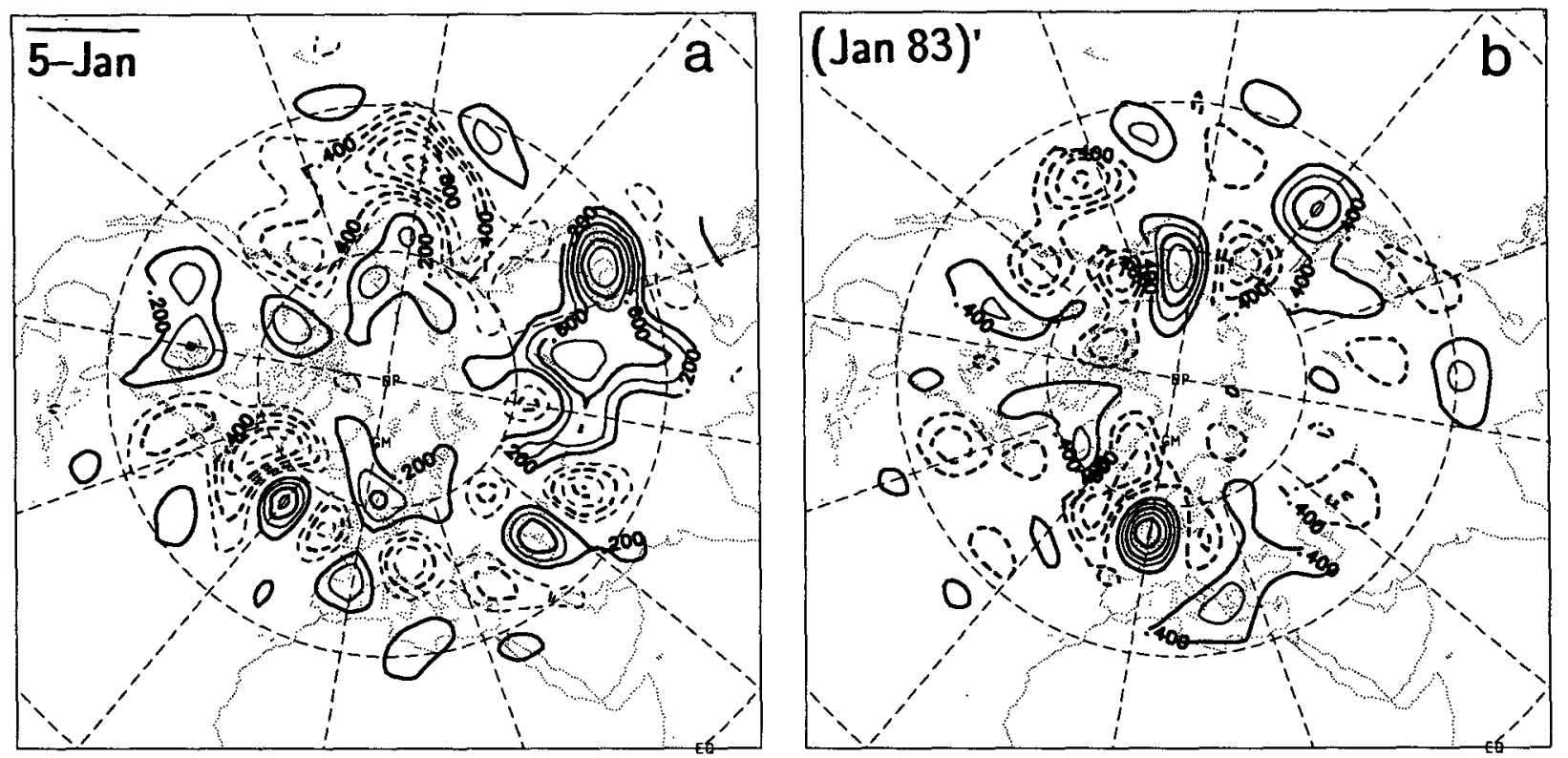

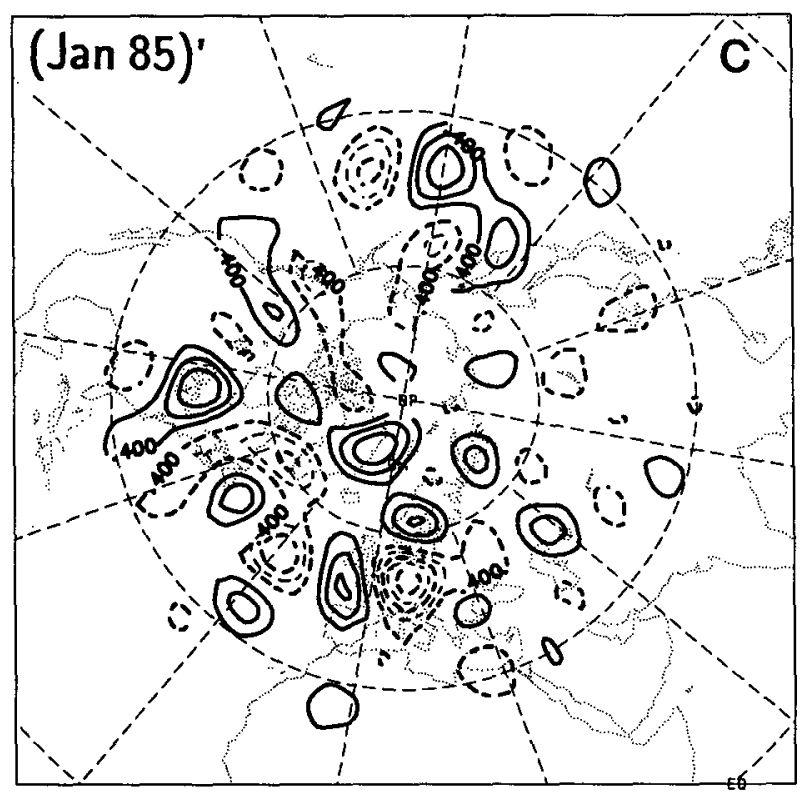

tation is increased, and may be traced directly to the noisy nature of our PV itself, even in the climate mean.

Application of the adjoint method to equivalent barotropic PV anomalies yields quite similar results to those obtained for tropospheric temperature in Part I. However, the adjoint "PV" (or information tracer) generally spreads out much more rapidly from its source during adjoint integration so that the forcing effects (and also the ICs) are more hemispheric in extent than in the $T$ case. This is a direct result of the higher advection speeds at upper levels and suggests the hemispheric nature (or otherwise) of the dynamics
FIG. 7. The forcing term, ${\overline{F Q^{*}}}^{I}$ from T42 experiments. (a) FiveJanuary mean (1981-85), contour interval 0.2 IDU QU day ${ }^{-1}$. (b) and (c): January 1983 and 1985 deviations from five-January mean, respectively, contour interval $0.4 \mathrm{IDU}$ QU day ${ }^{-1}$. Plotted fields have been truncated to T21; zero contour omitted. governing the regional anomalies (insofar as the monthly anomaly can be considered as a dynamical entity). Positive anomalies of equivalent barotropic PV are found to correspond to negative tropospheric temperature anomalies, with advection of anomalously cold air being mirrored by advection of anomalously high PV and vice versa. The largest $Q$ anomalies are primarily accounted for by anomalous ICs, with the implication that one month is insufficient time to generate important anomalies in the forcing term. Although the spatial distributions of the ICs look broadly similar in the T and PV cases, there are nevertheless 
some possibly important differences in preexisting source location, which are left to further study.

In contrast to the $T$ case in Part $I$, the effects of forcing on the target region are found to be negligible in the six-January mean, yet inter-January anomalies appear to be just as important. The climatological result is consistent with the better forward integration in the $Q$ case, and one would indeed expect $Q$ to be better conserved by the equivalent barotropic model, than temperature in the one-layer tropospheric model. The relatively small amplitudes of the forcing anomalies associated with the large $Q$ anomalies in January 1983 and 1985, together with the small-scale nature of their associated spatial distributions, makes it tempting to associate the forcing anomalies with noise. Indeed, we were unable to associate the forcing term with the prevalent weather regimes in January 1983 and 1985 (admitting that calendar months do not represent the ideal episodes to study). A more comprehensive study is needed to resolve this point.

The method is more sensitive to the dissipation than in the $T$ case, and higher horizontal resolution is required to ensure that the information tracer is well behaved (i.e., does not become negative), without dissipating important information. However, even at T42 a moderate dissipation must be used to avoid negative tracer values, and one cannot be sure that important information is not being dissipated. Flux correction techniques (Schmidt 1990) provide a means of avoiding negative tracer values altogether (by using a logarithmic transformation ), and these might be especially useful for longer time periods, perhaps also enabling lower resolution to be used.

The choice of variable in an adjoint study of regional anomalies will depend on the goal of the study. If the aim is to investigate the difference between GCM climates on a regional basis, with changes in the diabatic effects in mind, then temperature is probably the more suitable variable, despite the large adiabatic component in the forcing term in the one-layer temperature model. On the other hand, the PV has potential for studies of the dynamics of weather regimes on the weekly time scale.

As discussed in Part I, a simple adjoint tracer model "linearized" about the observed flow cannot tackle the nonlinear problem of disentangling the direct (advected) effect of remote forcing on the target region from the indirect effect that a forcing may have on the advecting flow itself. In addition, an advection model does not allow one to look farther upstream than advective length scales, though these are admittedly longer in the PV case than for tropospheric temperature. To address these limitations, we intend to apply the adjoint technique to a barotropic vorticity equation model, linearized about a ( say ) $300-\mathrm{mb}$ timemean flow. This would then determine whether remote Rossby wave source regions (say in the tropics) are implicated in midlatitude vorticity anomalies via barotropic Rossby wave propagation. The advective effects of the (linear) flow response to remote forcing might then also be examined using an adjoint advection model.

Acknowledgments. I thank M. Blackburn, J. Egger, W. Metz, and F. Schmidt for reading earlier versions of the manuscript and making many valuable suggestions. This work was supported by the Bavarian Climate Program.

\section{REFERENCES}

Blackmon, M. L., R. A. Madden, J. M. Wallace, and D. S. Gutzler, 1979: Geographical variations in the vertical structure of geopotential height fluctuations. J. Atmos. Sci., 36, 2450-2466.

Haynes, P. H., and M. E. McIntyre, 1987: On the evolution of vorticity and potential vorticity in the presence of diabatic heating and frictional or other forces. J. Atmos. Sci., 44, 828-841.

- , and -1990 : On the conservation and impermeability theorems for potential vorticity. J. Atmos. Sci., 47, 2021-2031.

Hoskins, B. J., M. E. McIntyre, and A. W. Robertson, 1985: On the use and significance of isentropic potential vorticity maps. Quart. J. Roy. Meteor. Soc., 111, 877-946.

Juckes, M. N., and M. E. McIntyre, 1987: A high-resolution onelayer model of breaking planetary waves in the stratosphere. Nature, 328, 590-596.

Marchuk, G. I., 1974: Numerical Solutions of Problems in Atmospheric and Oceanic Dynamics. Gidrometeoizdat Press (in Russian ).

McIntyre, M. E., and T. N. Palmer, 1984: The "surf zone" in the stratosphere. J. Atmos. Terr. Phys., 46, 825-600.

McWilliams, J. C., 1980: An application of equivalent modons to atmospheric blocking. Dyn. Atmos. Ocean, 5, 43-66.

Robertson, A. W., 1992: Diagnosis of regional monthly anomalies using the adjoint method. Part I: Temperature. J. Atmos. Sci., 49, 906-918.

Salby, M. L., 1989: Deep circulations under simple classes of stratification. Tellus, 41A, 48-65.

-, R. R. Garcia, D. O'Sullivan, and P. Callaghan, 1990: The interaction of horizontal eddy transport and thermal drive in the stratosphere. J. Atmos. Sci., 47, 1647-1665.

Schmidt, F., 1990: Flux correction by transformation of dependant variables. Beitr. Phys. Atmos., 63, 52-59. 